\title{
Iron L-edge absorption spectroscopy of iron pentacarbonyl and ferrocene in the gas phase
}

\author{
Kai Godehusen ${ }^{1}$, Tobias Richter ${ }^{2}$, Peter Zimmermann ${ }^{3}$, Philippe Wernet ${ }^{4}$
}

\author{
${ }^{1}$ Helmholtz-Zentrum Berlin, Hahn-Meitner-Platz 1, 14109 Berlin, Germany \\ ${ }^{2}$ European Spallation Source, Ole Maaløes Vej 3, 2200 Copenhagen, Denmark \\ ${ }^{3}$ Technische Universität Berlin, Hardenbergstraße 36, 10623 Berlin, Germany \\ ${ }^{4}$ Institute for Methods and Instrumentation for Synchrotron Radiation Research, Helmholtz- \\ Zentrum Berlin für Materialien und Energie GmbH, Albert-Einstein-Str. 15, 12489 Berlin, \\ Germany
}

\begin{abstract}
Fe L-edge x-ray absorption spectra of gas-phase iron pentacarbonyl and ferrocene measured in total-ion yield mode are reported. Comparison to previously published spectra of free iron atoms and gaseous iron chloride demonstrates how the interplay of local atomic multiplet effects and orbital interactions in the metal-ligand bonds varies for the different systems. We find changes in the degree of metal-ligand covalency to be reflected in the measured $2 p$ absorption onset. Orbital- or state-specific fragmentation is furthermore investigated in iron pentacarbonyl and ferrocene by analyzing the partial-ion-yield spectra at the Fe L-edge. Strong dependence of the yields of different fragments is observed in ferrocene in contrast to iron pentacarbonyl. This difference is attributed to the different degrees to which the $2 p$ core hole is screened in the two systems. We provide experimental benchmark spectra for new ab initio approaches for calculating metal L-edge absorption spectra of metal complexes.
\end{abstract}




\section{Introduction}

Iron pentacarbonyl $\left(\mathrm{Fe}(\mathrm{CO})_{5}\right)$ and ferrocene (bis( $n 5$-cyclopentadienyl)iron(II), denoted $\mathrm{Fe}(\mathrm{Cp})_{2}$ hereafter) have always attracted great interest for fundamental investigations of the electronic structure in coordination complexes due to the prototypical character of their metal-ligand bonds. They have also served as benchmark systems for new methods aiming at correlating chemical properties with new experimental observables. X-ray spectroscopy is well suited to study the electronic structure of $3 d$ transition-metal complexes due to its elemental and site specificity that enables addressing the bonds separately from the perspective of the metal center or the ligand [1-6]. X-ray absorption spectroscopy (XAS) in particular gives access to the unoccupied part of the valence electronic structure and hence is an established technique for the investigation of bonding in metal complexes. In the soft $x$-ray range, the most common method for addressing bonding at the metal site is XAS at the L-edge of $3 d$ transition-metals as it involves comparably strong dipole-allowed transitions of the metal $2 p$ electrons into the unoccupied orbitals thereby probing their $3 d$ character $[3,7-13]$. The spectra can furthermore be strongly influenced by local atomic multiplet effects in the coreexcited states [14]. Accounting for both the local atomic multiplets and the molecular valenceorbital interactions represents a challenge for current theories [7, 8, 11, 15-24]. Despite the general interest, Fe L-edge XAS of gas-phase $\mathrm{Fe}(\mathrm{CO})_{5}$ and $\mathrm{Fe}(\mathrm{CP})_{2}$ has not been reported yet to the best of our knowledge and it is the aim of the present investigation to fill this gap.

Wen, Rühl and Hitchcock reported in a seminal investigation the Fe L-edge inner-shell excitation spectra by electron energy loss (ISEELS) of $\mathrm{Fe}(\mathrm{CO})_{5}$ and $\mathrm{Fe}(\mathrm{Cp})_{2}$ in the gas phase [25]. As the spectra were measured in dipole scattering conditions, they should be comparable to the Fe L-edge absorption spectra from XAS. It is our aim here to complement the ISEELS data with Fe L-edge XAS.

Ion-yield XAS is an established method for determining the x-ray absorption spectrum of a gaseous sample. Total-ion-yield XAS displays the integrated intensities of all ions versus photon energy and directly corresponds to the absorption cross section if decay of the coreexcited states via fluorescence is negligible and if emission of neutral fragments can be omitted $[2,26]$. Both conditions are fulfilled for the investigations here, as the fluorescence yield is known to be very small in the range of L-edge absorption of $3 \mathrm{~d}$ transition-metals $(<0.1$ $\%$, [13]). Furthermore, core-excited state decay in $\mathrm{Fe}(\mathrm{CO})_{5}$ and $\mathrm{Fe}(\mathrm{Cp})_{2}$ is dominated by Auger decay leading to ionic fragments. This is evident from the investigations of Tamenori et al. who reported ion-yield XAS at the Fe M-edge of $\mathrm{Fe}(\mathrm{CO})_{5}$ and $\mathrm{Fe}(\mathrm{Cp})_{2}$ and of Heck et al. and Sistrunk et al. for ion-yield Fe M-edge XAS of $\mathrm{Fe}(\mathrm{CO})_{5}$ [27-30]. By resolving the nature of the various ionic fragments emitted upon x-ray irradiation, so-called partial-ion-yield spectra can be extracted. They contain information on orbital- or state-specific fragmentation of the probed sample [2, 26, 31-35].

Here we present total- and partial-ion yield XAS at the Fe L-edge of $\mathrm{Fe}(\mathrm{CO})_{5}$ and $\mathrm{Fe}(\mathrm{CP})_{2}$ in the gas phase. Extending earlier studies of Fe atoms and ionic Fe compounds in the gas phase [11] 
we now turn to systems with strong covalent interactions. By systematically investigating the partial-ion-yield spectra measured at the Fe L-edge of the two compounds we address the question in particular, whether the differences in metal-ligand bonding are expressed in the orbital-specific fragmentation patterns. This provides missing experimental information that as a benchmark for recently developed ab initio calculations treating both local atomic multiplet effects and valence-orbital interactions [12, 36-41]. Orbital-specific fragmentation measured for different $x$-ray resonances gives insight in particular into the nature of the corresponding core-excited states. The reported Fe L-edge absorption spectra of gas-phase $\mathrm{Fe}(\mathrm{CO})_{5}$ finally complement recent steady-state [12] and time-resolved [42, 43] investigations of $\mathrm{Fe}(\mathrm{CO})_{5}$ in solution with resonant inelastic x-ray scattering and hard $\mathrm{x}$-ray spectroscopy investigations of $\mathrm{Fe}(\mathrm{CO})_{5}$ and $\mathrm{Fe}(\mathrm{Cp})_{2}$ at the Fe K-edge $[1,4-6]$.

\section{Experimental}

The Fe compounds were purchased from Sigma-Aldrich and used without further purification. They were introduced into the interaction region via a small gas nozzle. In the case of $\mathrm{Fe}(\mathrm{Cp})_{2}$ the whole gas inlet system, containing the solid sample was heated to about $40^{\circ} \mathrm{C}$ in order to reach a sufficient gas pressure. Special care was taken to keep the $\mathrm{Fe}(\mathrm{CO})_{5}$ sample in complete darkness to prevent the sample from decomposition by photochemical reactions. Since the measurements have been repeated over several days without any sign of change in the spectral features, we are certain that our spectra reflect pure $\mathrm{Fe}(\mathrm{CO})_{5}$.

The measurements were carried out at the UE52-SGM beamline at the electron storage ring BESSY II in Berlin. The photon energy bandwidth amounted to 0.2-0.3 eV for the reported spectra. The measured linewidth for all spectra is on the order of $0.8 \mathrm{eV}$ and is mainly given by the natural linewidth of the $2 p$ core-excited XAS final states as determined by the $2 p$ corehole lifetime and by inhomogeneous broadening. A time of flight ion spectrometer (TOF) was used for recording the ion-yield spectra and the TOF was operated in pulsed mode. The acquisition time for the displayed data typically amounted to 3 hours.

We are not giving absolute numbers on the branching ratios into the different fragmentation channels since the TOF measurements are always affected by the different detection probabilities for ions of different masses.

The photon energy calibration is based on measurements of Xe noble gas samples with wellknown resonance energies [44]. This procedure resulted in an uncertainty of the absolute values of the photon energies in the reported spectra of $0.1 \mathrm{eV}$. The uncertainty when comparing photon energies in the $\mathrm{Fe}(\mathrm{CO})_{5}$ and $\mathrm{Fe}(\mathrm{Cp})_{2}$ spectra is even below that.

\section{Results and discussion}


The total-ion-yield Fe L-edge absorption spectra of gaseous $\mathrm{Fe}(\mathrm{CO})_{5}$ and $\mathrm{Fe}(\mathrm{Cp})_{2}$ are compared to the previously published spectra of gaseous $\mathrm{FeCl}_{2}$ and free $\mathrm{Fe}$ atoms [11] in Figure 1. The spectra of $\mathrm{Fe}(\mathrm{CO})_{5}$ and $\mathrm{Fe}(\mathrm{Cp})_{2}$ are qualitatively very similar to the Fe L-edge inner-shell excitation spectra taken with ISEELS by Wen et al. [25] underlining the close correspondence of ISEELS in dipole scattering conditions and XAS. Comparison of our $\mathrm{Fe}(\mathrm{CO})_{5}$ and $\mathrm{Fe}(\mathrm{Cp})_{2} \mathrm{X}-$ ray absorption spectra with $\mathrm{FeCl}_{2}$ and $\mathrm{Fe}$ atoms with our accurately calibrated photon-energy axis and the partial-ion yield absorption spectra (following figures) reveal new information compared to the state of the art defined by the ISEELS spectra. The spectra in Figure 1 reflect the varying contributions of local atomic multiplet effects and orbital interactions arising from metal-ligand bonding. Trends of general interest are readily visible. The spectra apparently change from exhibiting numerous comparably sharp lines in free Fe atoms to broad peaks in $\mathrm{Fe}(\mathrm{CO})_{5}$ and $\mathrm{Fe}(\mathrm{Cp})_{2}$. The spectrum of free Fe atoms is solely determined by the local atomic multiplet effects and the corresponding spectral features are largely preserved in $\mathrm{FeCl}_{2}$ albeit somewhat broadened [11]. This reflects the ionic bonding in $\mathrm{FeCl}_{2}$ with the multiplet components of the ionic Fe center $[9,11]$. The comparably strong covalent interactions in $\mathrm{Fe}(\mathrm{CO})_{5}$ and $\mathrm{Fe}(\mathrm{Cp})_{2}$ in contrast explain the considerable broadening of the peaks. This increase of covalent interactions is also visible in the onset of the x-ray absorption spectra. The L-edge absorption onset corresponds to the lowest $2 p \rightarrow 3 d$ excitation energy in the spectrum and it apparently increases from approximately $705 \mathrm{eV}$ in free Fe atoms to $706 \mathrm{eV}$ in $\mathrm{FeCl}_{2}$ and to $707.5 \mathrm{eV}$ in $\mathrm{Fe}(\mathrm{Cp})_{2}$ and $\mathrm{Fe}(\mathrm{CO})_{5}$ with no notable difference between $\mathrm{Fe}(\mathrm{Cp})_{2}$ and $\mathrm{Fe}(\mathrm{CO})_{5}$ (Figure 1). The shift of this onset results from changes in screening of the $2 \mathrm{p}$ core hole in the final (core-excited) states in the systems. In a qualitative and approximate picture the shift to higher energies can be interpreted to reflect an increase of the local (positive) charge at the Fe center with a concomitant decrease of the ability to screen the core hole. This has been analyzed before in numerous investigations such as in $3 \mathrm{~d}$ transitions metals clusters as a function of cluster size [22] and it is related to the chemical concept of increasing nominal oxidation state of the metal center in coordination compounds and the correspondingly increasing L-edge absorption onset [45]. With increasing covalent interactions when going from free Fe atoms to the covalently bonded $\mathrm{Fe}(\mathrm{Cp})_{2}$ and $\mathrm{Fe}(\mathrm{CO})_{5}$ the local charge at Fe decreases as it delocalizes to the ligands. This reduces the screening ability at the Fe center as the effective nuclear charge on Fe increases. In turn, the L-edge absorption onset shifts to higher photon energy.

Our measured photon energies for the four main resonances in the L-edge absorption spectra of $\mathrm{Fe}(\mathrm{CP})_{2}$ and $\mathrm{Fe}(\mathrm{CO})_{5}$ (two resonance at each the $\mathrm{L}_{3}$ and the $\mathrm{L}_{2}$ edge) are summarized in Table 1. It has to be noted that our resonance energies differ from the values reported by Wen et al. by up to $1.5 \mathrm{eV}$. We note that the values for absolute photon energies in our spectra have an uncertainty of $0.1 \mathrm{eV}$. The Fe L-edge absorption spectra of $\mathrm{Fe}(\mathrm{CO})_{5}$ and $\mathrm{Fe}(\mathrm{Cp})_{2}$ were calculated before by Fronzoni et al. [16] and a qualitative match with the measured spectra from Wen et al. [25] was achieved. Based on this and the former study by Wen et al. [25] we also summarize the assignments of the resonances to orbital excitations in Table 1. In both $\mathrm{Fe}(\mathrm{Cp})_{2}$ and in $\mathrm{Fe}(\mathrm{CO})_{5}$ the first resonance at each the $\mathrm{L}_{3}$ and the $\mathrm{L}_{2}$ edge corresponds to 
excitations of the Fe $2 p$ electrons into Fe-centered molecular orbitals of mainly $3 d$ character. The second resonances, in contrast, correspond to excitations of the Fe $2 p$ electrons into ligand-centered molecular orbitals of mainly $\pi^{*}$ character. The question occurs, whether this difference in the nature of the orbital excitations is reflected in the partial-ion-yield absorption spectra. Based on a simple notion one could expect that $x$-ray absorption in the second resonance with $2 p$ excitation into ligand orbitals leads to more efficient fragmentation of the molecule into the metal and ligand fragments or into fragmented ligands. It is the purpose of the following discussion to test this notion with the partial-ion yield absorption spectra.

We show time-of-flight spectra of $\mathrm{Fe}(\mathrm{CO})_{5}$ in Figure 2. Peaks assigned to $\mathrm{N}^{+}, \mathrm{CO}^{2+}, \mathrm{Ne}^{+}$and $\mathrm{CO}_{2}{ }^{+}$ are attributed to ionization of rest gas in the chamber. The comparison of time-of-flight spectra taken with photon energies above and below the Fe L-absorption edge allows identifying which fragment peak stems from element- or Fe-site specific x-ray induced fragmentation. It also allows determining how strongly these peaks are resonantly enhanced (Figure 2, top panel). Fragments are assigned to peaks in the difference spectrum displayed in the bottom panel of Figure 2 (intensities above minus intensities below the resonance). Strikingly, we do not observe any un-fragmented charged (singly ionized, e.g.) Fe(CO) 5 molecules as we have no evidence for intensity in the region around TOF channel 1260 where we would expect peaks assigned to $\mathrm{Fe}(\mathrm{CO})_{5}{ }^{+}$. In fact, we do not observe any large fragments. The largest fragment we detect is $\mathrm{Fe}(\mathrm{CO})_{2}{ }^{+}$(we indicated the alternative assignment of the $\mathrm{Fe}(\mathrm{CO})_{2}{ }^{+}$peak as $\mathrm{Fe}(\mathrm{CO})_{4}{ }^{2+}$, two fragments we cannot distinguish, but note that the former assignment to a singly charged ion is more likely to be correct). We observe a strong signal assigned to $\mathrm{Fe}^{+}$and see some contribution of intact ionized $\mathrm{CO}^{+}$fragments, but no $\mathrm{C}$ or $\mathrm{O}$ ions are detected. Hence in the photo-excitation of $\mathrm{Fe}(\mathrm{CO})_{5}$ above the Fe L-edge, fragmentation apparently dominates over ionization and the fragmentation seems to be very effective.

The partial-ion yield absorption spectra of $\mathrm{Fe}(\mathrm{CO})_{5}$ in Figure 3 show how the various fragmentation channels couple to the various resonances in the spectrum. Notably, the six partial-ion yield spectra of the six strongest fragments in the time-of-flight spectrum are very similar demonstrating that the coupling of all fragmentation channels to the $x$-ray resonances is equally efficient. This lack of state-specific fragmentation shows that no matter in which molecular orbital the Fe $2 p$ electrons are excited into, the molecular fragmentation is equally probable and leads to similar distributions of fragments. There are a number of possible explanations for this observation. It could indicate that the assignments of Table 1 are incomplete or too approximate in the sense that the core-excited states are strongly mixed and all have strong Fe $3 d$ and ligand $\pi^{*}$ character. The ab initio calculations reported in [12] and [43] confirm this notion. It could furthermore indicate that all core-excited states are highly dissociative no matter in which orbital the excitation proceeds. Future calculations of the nature of the core-excited states of $\mathrm{Fe}(\mathrm{CO})_{5}$ could give more insight into this. For the moment while such calculations are not available, investigation of $\mathrm{Fe}(\mathrm{Cp})_{2}$ and the comparative discussion further below will yield more insight into these arguments. 
The time-of-flight spectra of $\mathrm{Fe}(\mathrm{Cp})_{2}$ for excitation above and below the Fe L-absorption resonance are displayed in Figure 4. The Xe peaks (Figure 4, top panel) again stem from ionization of Xe rest gas in the chamber used for photon-energy calibration. Assignments of the peaks to $\mathrm{Fe}(\mathrm{Cp})_{2}$ fragments is assisted by resonant enhancement with the difference spectrum in the bottom panel of Figure 4. We first note that we detect a strong peak associated with the singly charged un-fragmented molecule $\mathrm{Fe}(\mathrm{Cp})_{2}{ }^{+}$showing that ionization with respect to fragmentation is much stronger in $\mathrm{Fe}(\mathrm{Cp})_{2}$ compared to $\mathrm{Fe}(\mathrm{CO})_{5}$. Furthermore, we also detect strong signals from large fragments and the largest fragment is $\mathrm{Fe}(\mathrm{Cp})^{+}$where one $\mathrm{Cp}$ ring is missing. We indeed also observe the corresponding signal from singly charged $\mathrm{Cp}$ rings $\left(\mathrm{Cp}^{+}\right)$. As for $\mathrm{Fe}(\mathrm{CO})_{5}$ we see a strong $\mathrm{Fe}^{+}$signal. In contrast to $\mathrm{Fe}(\mathrm{CO})_{5}$ we do observe signal from fragmented ligands in the case of $\mathrm{Fe}(\mathrm{Cp})_{2}$ (fragmented rings such as $\mathrm{C}_{n} \mathrm{H}_{m}{ }^{+}(n=1$, 2), $\mathrm{C}_{3} \mathrm{H}_{\mathrm{m}}{ }^{+}$). Apparently, fragmentation of the molecule into Fe and intact ligands is less efficient in $\mathrm{Fe}(\mathrm{Cp})_{2}$ compared to $\mathrm{Fe}(\mathrm{CO})_{5}$ while fragmentation of the ligands themselves is more efficient in $\mathrm{Fe}(\mathrm{Cp})_{2}$ compared to $\mathrm{Fe}(\mathrm{CO})_{5}$.

The partial-ion yield absorption spectra of $\mathrm{Fe}(\mathrm{Cp})_{2}$ in Figure 5 now exhibit a remarkable dependence of the fragment yield on the x-ray resonances. This fragment-specificity clearly reflects orbital-specific fragmentation of $\mathrm{Fe}(\mathrm{CP})_{2}$ at the Fe L-edge. Note that the normalized spectra in Figure 5 allow interpretation of relative intensities only as the spectra are not weighted with the $x$-ray absorption cross section and the relative intensities of the different fragmentation channels cannot be deduced from our spectra. The first resonance at $709 \mathrm{eV}$ is more intense than the second resonance at $712.1 \mathrm{eV}$ in the partial-ion yield spectra of $\mathrm{Fe}(\mathrm{CP}) 2_{2}{ }^{+}$ and $\mathrm{Fe}(\mathrm{Cp})_{2}{ }^{2+}$. This indicates that Fe $2 \mathrm{p}$ excitation in $\mathrm{Fe}(\mathrm{Cp})_{2}$ into the Fe-centered molecular orbitals of predominantly $3 \mathrm{~d}$ character leads to more efficient ionization of the molecule without fragmentation compared to excitation at the second resonance. This could be related to a stronger bonding character of the orbitals excited to in the first resonance compared to the second resonance and compared to $\mathrm{Fe}(\mathrm{CO})_{5}$. The second resonance is more intense than the first for completely fragmented ions with or without $\mathrm{Fe}\left(\mathrm{C}_{n} \mathrm{H}_{\mathrm{m}}{ }^{+}, \mathrm{C}_{3} \mathrm{H}_{\mathrm{m}}{ }^{+}, \mathrm{FeC}_{2} \mathrm{H}_{\mathrm{m}}{ }^{+}, \mathrm{FeC}_{8} \mathrm{H}_{\mathrm{m}}{ }^{2+}\right)$ indicating that Fe $2 p$ excitation into the ligand-centered $\pi^{*}$ orbitals that is delocalized over the whole $\mathrm{Cp}$ ring leads to more efficient fragmentation than excitation at the first resonance. This could be related to a stronger anti-bonding character of the orbitals excited to in the second resonance compared to the first. First and second resonances are approximately equally strong for $\mathrm{Fe}^{+}, \mathrm{Cp}^{+}$, and $\mathrm{FeCp}^{+}$demonstrating that both resonances equally efficient couple to abstraction of one or both of the $\mathrm{Cp}$ rings from $\mathrm{Fe}(\mathrm{Cp})_{2}$. We also note that in the spectra in Figure 5 the two resonances at the $L_{2}$ edge are approximately equally intense for all fragments. It is known that the core-excited states at the $L_{2}$ absorption edge of $3 d$ transitionmetal atoms are often more strongly mixed $[14,19,21]$. Here, this lack of fragment- or orbitalspecificity could thus be explained by strong mixing of Fe $3 d$ and ligand $\pi^{*}$ character of the core-excited states at the $L_{2}$ edge.

Combining all experimental observations and discussions allows some general statements to be made. For both $\mathrm{Fe}(\mathrm{CO})_{5}$ and $\mathrm{Fe}(\mathrm{Cp})_{2}$ anti-bonding orbitals are populated at the Fe L-edges but the extent to which this excitation leads to ionization of the intact molecule versus 
fragmentation varies with the x-ray excitation energy. That we do not observe any charged but intact $\mathrm{Fe}(\mathrm{CO})_{5}$ molecules in contrast to $\mathrm{Fe}(\mathrm{Cp})_{2}$ and that fragmentation is strongly orbitalspecific in $\mathrm{Fe}(\mathrm{Cp})_{2}$ in contrast to $\mathrm{Fe}(\mathrm{CO})_{5}$ indicates fundamental differences in the fragmentation pathways and efficiencies of the two molecules. Although the first resonance in $\mathrm{Fe}(\mathrm{CO})_{5}$ can be assigned to Fe-centered orbitals with $\mathrm{Fe} 3 \mathrm{~d}$ character as in $\mathrm{Fe}(\mathrm{CP})_{2}$ we do not observe any charged intact $\mathrm{Fe}(\mathrm{CO})_{5}$. This indicates that the core-hole in $\mathrm{Fe}(\mathrm{CO})_{5}$ even for this comparably local excitation is not well screened making the core-excited state dissociative and leading to fragmentation instead of ionization. In $\mathrm{Fe}(\mathrm{Cp})_{2}$ in contrast screening of the corehole in the comparably local excitation into Fe-centered orbitals with $3 \mathrm{~d}$ character by the delocalized electron density in the two $\mathrm{Cp}$ rings does not destabilize the molecule hence favoring ionization over fragmentation. This picture fits into how strong fragmentation is compared to ionization in the two systems for other photon energies. At the Fe M-absorption edge Tamenori et al. found signals from charged intact molecules for both $\mathrm{Fe}(\mathrm{CO})_{5}$ and $\mathrm{Fe}(\mathrm{Cp})_{2}$ but the $\mathrm{Fe}(\mathrm{Cp})^{+}$signal was considerably stronger than the $\mathrm{Fe}(\mathrm{CO})_{5}{ }^{+}$signal compared to the fragment signals of the respective systems $[29,30]$ indicating as well less efficient core-hole screening in $\mathrm{Fe}(\mathrm{CO})_{5}$ compared to $\mathrm{Fe}(\mathrm{Cp})_{2}$ at the M-edge. Finally, $\mathrm{Fe}(\mathrm{CO})_{5}$ in contrast to $\mathrm{Fe}(\mathrm{Cp})_{2}$ is very sensitive to visible light and temperature and comparably easily decomposes upon optical or thermal excitation. Excitation of $\mathrm{Fe}(\mathrm{Cp})_{2}$ at the second resonance leads to a very strong $\mathrm{C}_{n} \mathrm{H}_{m}{ }^{+}(n=1,2)$ signal of a completely fragmented ligand. With our approach we cannot determine whether the $\mathrm{Fe}(\mathrm{Cp})^{+}$signal also observed stems from the same fragmentation channel. Assuming it does, it could indicate that promotion of an Fe $2 p$ electron into the ligand-centered anti-bonding $\pi^{*}$ orbitals in $\mathrm{Fe}(\mathrm{Cp})_{2}$ at the second resonance destabilizes one of the $\mathrm{Cp}$ rings leading to its dissociation (and subsequent fragmentation) while leaving $\mathrm{FeCp}$ behind. This could indicate that ligands in $\mathrm{Fe}(\mathrm{Cp})_{2}$ can be selectively excited. In $\mathrm{Fe}(\mathrm{CO})_{5}$ in contrast and in terms of Fe $2 p$ photo-excitation the $\mathrm{CO}$ ligands seem to be more equivalent and hence seem to be coupled in a sense that Fe $2 p$ excitation always and no matter which resonance is chosen leads to small fragments with at most one CO remaining on the Fe.

\section{Summary and conclusions}

We report Fe L-edge $\mathrm{x}$-ray absorption spectra of gas-phase iron pentacarbonyl $\left(\mathrm{Fe}(\mathrm{CO})_{5}\right)$ and ferrocene $\left(\mathrm{Fe}(\mathrm{CP})_{2}\right)$. The spectra were measured by detecting the total ion-yield versus photon energy and complement the state of the art Fe L-edge spectra taken with inner-shell excitation by electron energy loss from more than 20 years ago. With our accurately calibrated photon energy and the comparison of our x-ray absorption spectra of $\mathrm{Fe}(\mathrm{CO})_{5}$ and $\mathrm{Fe}(\mathrm{Cp})_{2}$ with the previously reported spectra of free $\mathrm{Fe}$ atoms and gaseous $\mathrm{FeCl}_{2}$ we correlate experimental observables in the L-edge absorption spectra with metal-ligand bonding. We address in particular how the interplay of local atomic multiplet effects and orbital interactions in the metal-ligand bonds varies for the different systems. Confirming established notions we find changes in the degree of metal-ligand covalency and the related changes in the local charge at the Fe center or, in other words, of the nominal oxidation state, to be reflected in the 
measured $2 p$ absorption onset. We furthermore report orbital- or state-specific fragmentation partial-ion-yield $x$-ray absorption spectra of $\mathrm{Fe}(\mathrm{CO})_{5}$ and $\mathrm{Fe}(\mathrm{Cp})_{2}$. Our measurements at the Fe L-edge complement and extend earlier partial-ion yield $x$-ray absorption investigations at the Fe M-edge. We test the notion whether differences in the metal-ligand bonds are expressed in the partial ion yields and find strong state- or orbitalspecific fragmentation in $\mathrm{Fe}(\mathrm{Cp})_{2}$. In $\mathrm{Fe}(\mathrm{CO})_{5}$, in contrast, this strong dependence is not found. This is attributed to the different degrees to which the $2 p$ core hole can be screened in the two systems. The electron-rich $\mathrm{Cp}$-ring ligands are found to more efficiently screen the $2 p$ hole for excitations into local Fe-centered molecular orbitals with strong $3 \mathrm{~d}$ character. We provide experimental benchmark spectra for new ab initio approaches for calculating metal L-edge absorption spectra of metal complexes. Our experimental data demonstrate that besides the nature of the metal-ligand bonds the nature of the $2 p$ core-excited states has to be correctly described to adequately reproduce the observed orbital-specific fragmentation yields at the Fe L-absorption edge.

\section{Acknowledgements}

We gratefully acknowledge the continuous support by the BESSY II staff and we thank HZB for the allocation of synchrotron radiation beamtime. 
Table 1: Experimentally determined photon energies as determined here for the main resonances in the total-ion-yield Fe L-edge absorption spectra of $\mathrm{Fe}(\mathrm{CO})_{5}$ and $\mathrm{Fe}(\mathrm{Cp})_{2}$ and assignments to the final molecular orbitals for the respective $2 p$ excitations (from ref. 25 ).

\begin{tabular}{|c|c|c|c|}
\hline \multicolumn{2}{|c|}{$\mathrm{Fe}(\mathrm{CO})_{5}$} & \multicolumn{2}{|c|}{$\mathrm{Fe}(\mathrm{Cp})_{2}$} \\
\hline Photon energy (eV) & Assignment & Photon energy $(\mathrm{eV})$ & Assignment \\
\hline \multicolumn{2}{|l|}{$\mathrm{L}_{3}$} & \multicolumn{2}{|c|}{$\mathrm{L}_{3}$} \\
\hline 708.5 & Fe $3 d$ & 709.5 & $4 e_{1 g}$ (-like), Fe $3 d$ \\
\hline 710.5 & $\pi^{*}(\mathrm{CO})$ & 712.1 & $3 e_{2 u}$ (-like), Сp $\pi^{*}$ \\
\hline \multicolumn{2}{|l|}{$\mathbf{L}_{2}$} & \multicolumn{2}{|c|}{$\mathbf{L}_{2}$} \\
\hline 720.8 & $\mathrm{Fe} 3 d$ & 721.9 & $4 e_{1 g}$ (-like), Fe $3 d$ \\
\hline 723.1 & $\pi^{*}(\mathrm{CO})$ & 724.7 & $3 e_{2 u}$ (-like), Сp $\pi^{*}$ \\
\hline
\end{tabular}




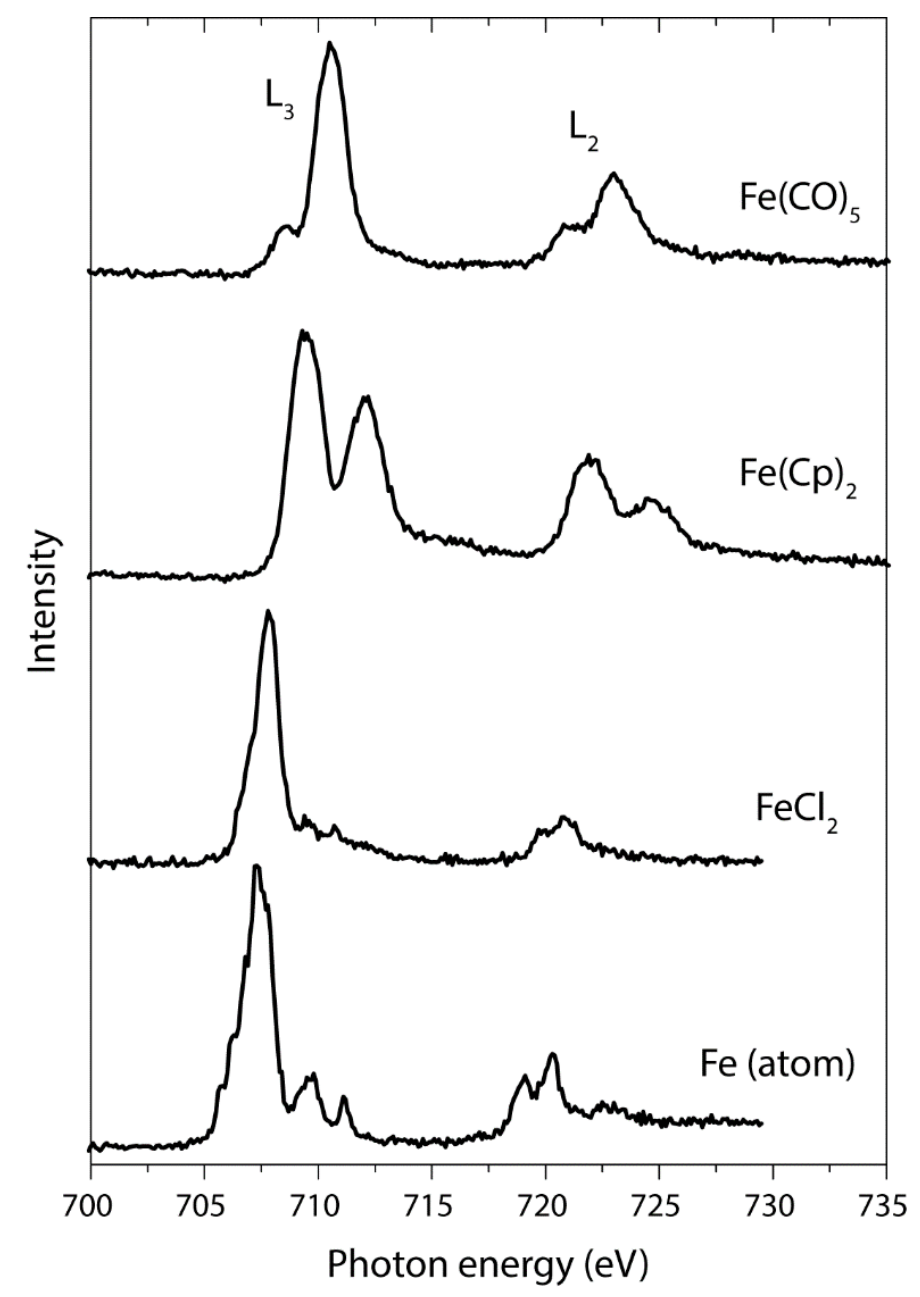

Figure 1: Total-ion-yield Fe L-edge x-ray absorption spectra of ironpentacarbonyl $\left(\mathrm{Fe}(\mathrm{CO})_{5}\right)$, ferrocene $\left(\mathrm{Fe}(\mathrm{Cp})_{2}\right)$, iron chloride $\left(\mathrm{FeCl}_{2}\right)$, and atomic $\mathrm{Fe}$ in the gas phase (the spectra of $\mathrm{FeCl}_{2}$ and Fe taken from ref. 11). 


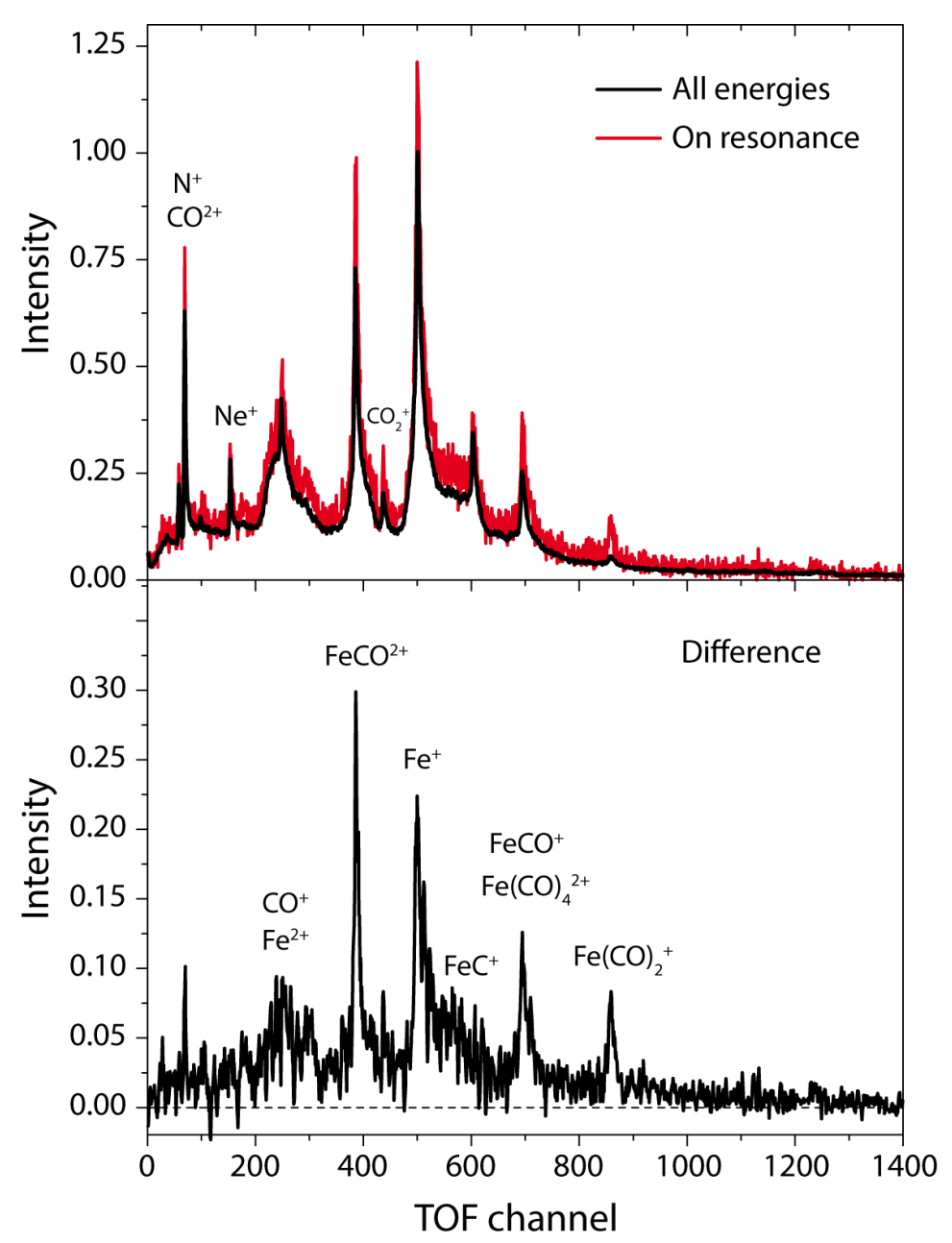

Figure 2: Time-of-flight spectra of iron pentacarbonyl $\left(\mathrm{FeCO}_{5}\right)$. Top panel: Sum of spectra taken over the whole Fe L-edge (photon energies between $700 \mathrm{eV}$ and $735 \mathrm{e}$, black) and sum of spectra taken over the $L_{3}$ edge only (red). Intensities are normalized to one at maximum in the spectrum taken over the whole photon energy range. Bottom panel: Difference of the spectra in the top panel with assignments to fragmentations products. (Multible products are given where the assignment is ambiguous). 


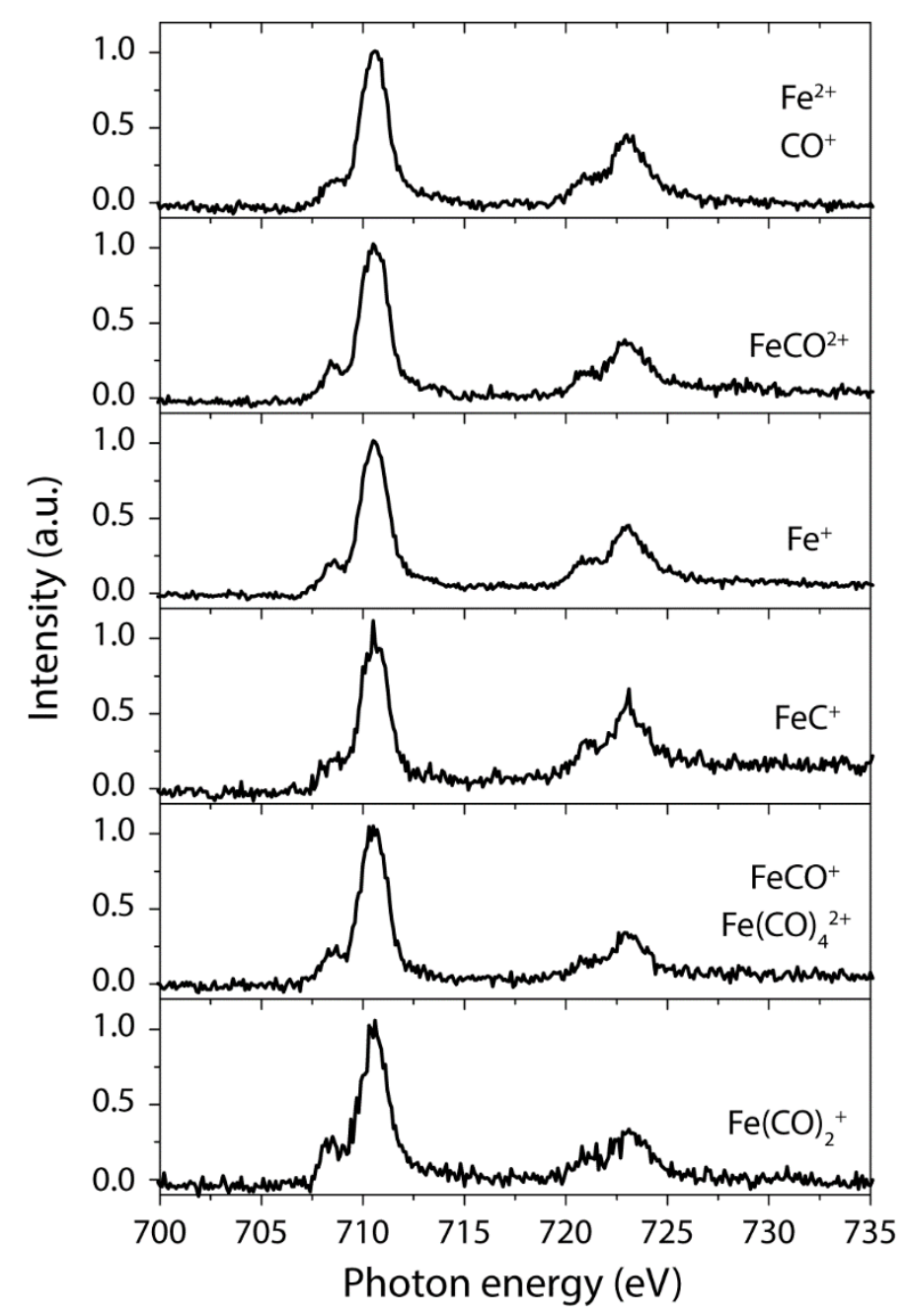

Figure 3: Partial-ion-yield Fe L-edge x-ray absorption spectra of ironpentacarbonyl $\left(\mathrm{Fe}(\mathrm{CO})_{5}\right)$ displaying the photon-energy dependence of the intensity of the main fragmentations products (see Figure 2). All spectra are normalized to one at their maximum. 


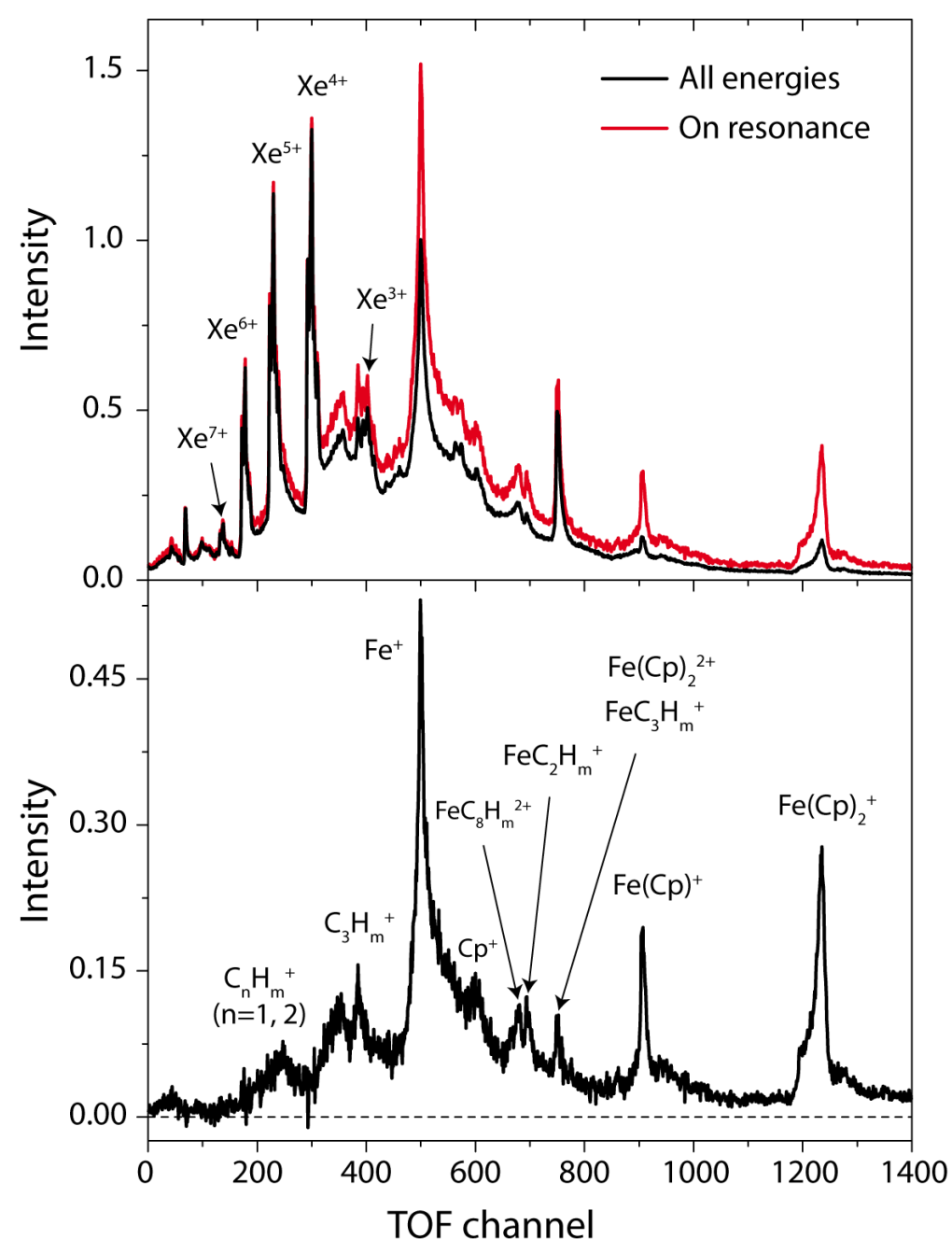

Figure 4: Time-of-flight-spectra of iron ferrocene $\left(\mathrm{Fe}(\mathrm{Cp})_{2}\right)$. Top panel: Sum of spectra taken over the whole Fe L-edge (photon energies between $700 \mathrm{eV}$ and $735 \mathrm{e}$, black) and sum of spectra taken over the $\mathrm{L}_{3}$ edge only (red). Intensities are normalized to one at the $\mathrm{Fe}^{+}$peak in the spectrum taken over the whole photon energy range. Bottom panel: Difference of the spectra in the top panel with assignments to fragmentations products (multiple products are given where the assignment is ambiguous). 


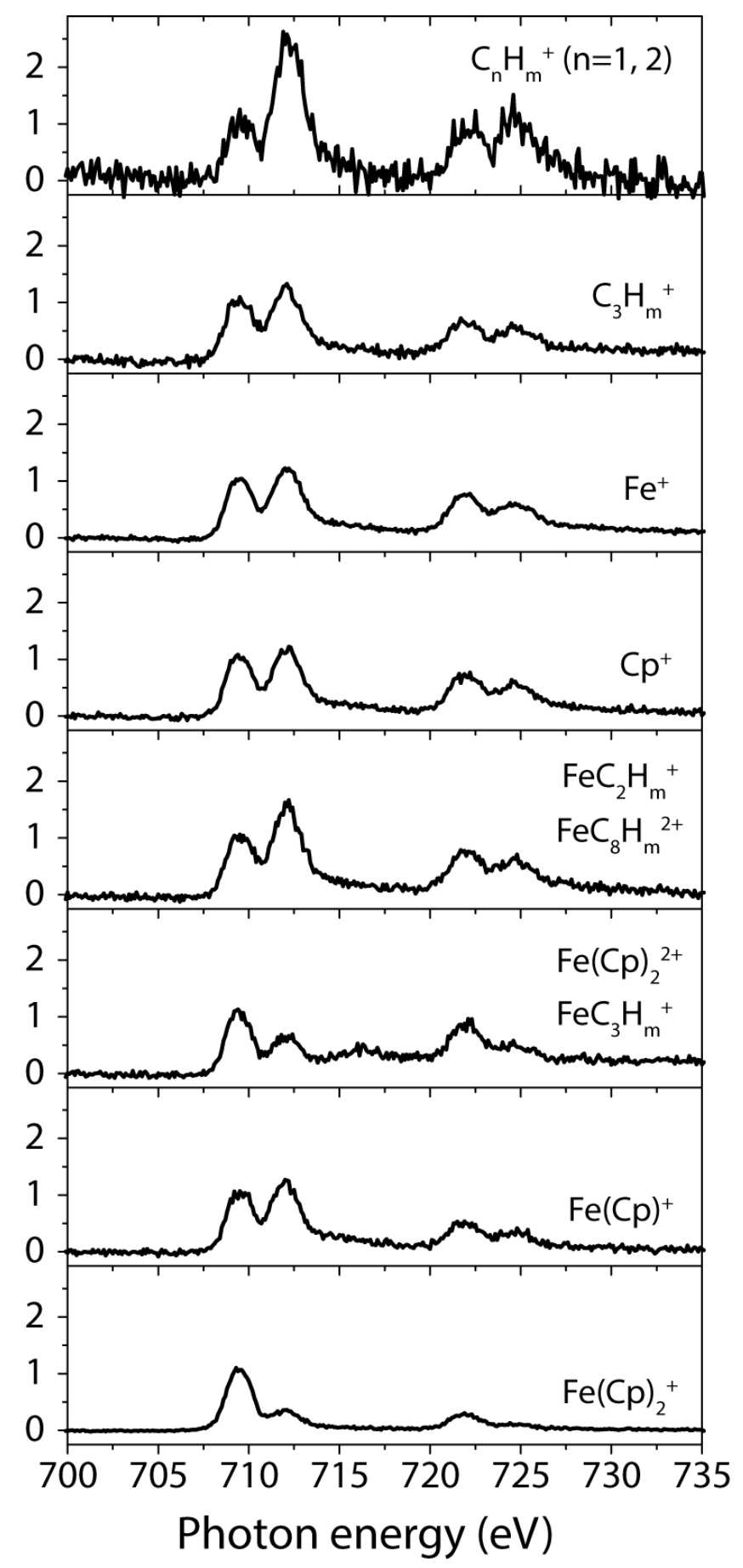

Figure 5: Partial-ion-yield Fe L-edge x-ray absorption spectra of ferrocene $\left(\mathrm{Fe}(\mathrm{Cp})_{2}\right)$ displaying the photon-energy dependence of the intensity of the main fragmentations products (see Figure 4). All spectra are normalized to one at their first (low-energy) peak. 


\section{References}

1. Atkins, A.J., M. Bauer, and C.R. Jacob, High-resolution X-ray absorption spectroscopy of iron carbonyl complexes. Phys Chem Chem Phys, 2015. 17(21): p. 13937-48.

2. Eberhardt, W., CORE ELECTRON EXCITATIONS AND DECAY IN MOLECULES. Physica Scripta, 1987. T17: p. 28-38.

3. Kunnus, K., et al., Viewing the Valence Electronic Structure of Ferric and Ferrous Hexacyanide in Solution from the Fe and Cyanide Perspectives. J Phys Chem B, 2016. 120(29): p. 7182-94.

4. Lancaster, K.M., K.D. Finkelstein, and S. DeBeer, K beta X-ray Emission Spectroscopy Offers Unique Chemical Bonding Insights: Revisiting the Electronic Structure of Ferrocene. Inorganic Chemistry, 2011. 50(14): p. 6767-6774.

5. Atkins, A.J., M. Bauer, and C.R. Jacob, The chemical sensitivity of X-ray spectroscopy: high energy resolution XANES versus $X$-ray emission spectroscopy of substituted ferrocenes. Phys Chem Chem Phys, 2013. 15(21): p. 8095-105.

6. Pollock, C.J. and S. DeBeer, Insights into the geometric and electronic structure of transition metal centers from valence-to-core X-ray emission spectroscopy. Acc Chem Res, 2015. 48(11): p. 2967-75.

7. Hatsui, T. and N. Kosugi, Metal-to-ligand charge transfer in polarized metal L-edge X-ray absorption of $\mathrm{Ni}$ and $\mathrm{Cu}$ complexes. Journal of Electron Spectroscopy and Related Phenomena, 2004. 136(1-2): p. 67-75.

8. Hatsui, T., et al., Ni $2 p$ excitation spectra of some planar Ni complexes. Journal of Electron Spectroscopy and Related Phenomena, 1998. 88-91: p. 45-409.

9. Hirsch, K., et al., 2px-ray absorption of free transition-metal cations across the 3dtransition elements: Calcium through copper. Physical Review A, 2012. 85(6).

10. Mitzner, R., et al., L-Edge X-ray Absorption Spectroscopy of Dilute Systems Relevant to Metalloproteins Using an X-ray Free-Electron Laser. J Phys Chem Lett, 2013. 4(21): p. 36413647.

11. Richter, T., et al., Interplay of intra-atomic and interatomic effects: An investigation of the $2 p$ core level spectra of atomic Fe and molecular FeCl2. Physical Review Letters, 2004. 93(2).

12. Suljoti, E., et al., Direct Observation of Molecular Mixing in a Solvated Organometallic Complex. Angewandet Chemie, International Edition, 2013. 52: p. 9841-9844.

13. Wernet, P., et al., Dissecting Local Atomic and Intermolecular Interactions of Transition-Metal Ions in Solution with Selective X-ray Spectroscopy. J Phys Chem Lett, 2012. 3(23): p. 3448-53.

14. Martins, M., et al., Open shells and multi-electron interactions: core level photoionization of the $3 d$ metal atoms. Journal of Physics B: Atomic, Molecular and Optical Physics, 2006. 39(5): p. R79-R125.

15. Bagus, P.S., et al., Atomic many-body effects for the p-shell photoelectron spectra of transition metals. Physical Review Letters, 2000. 84(10): p. 2259-2262.

16. Fronzoni, G., et al., 2p-]3d Excitations in Transition-Metal Compounds - a Computational Investigation on $\mathrm{Fe}(\mathrm{Co}) 5, \mathrm{Fe}(\mathrm{C5h} 5) 2$ and $\mathrm{Cr}(\mathrm{Co}) 6$. Journal of Electron Spectroscopy and Related Phenomena, 1993. 62(3): p. 245-262.

17. Godehusen, K., et al., $2 p$ photoionization of atomic Ni: A comparison with Ni metal and NiO photoionization. Physical Review Letters, 2002. 88(21).

18. Taguchi, M., T. Uozumi, and A. Kotani, Theory of X-ray photoemission and X-ray emission spectra in Mn compounds. Journal of the Physical Society of Japan, 1997. 66(1): p. 247-256.

19. Wernet, P., et al., $2 p$ photoelectron spectra and linear alignment dichroism of atomic Cr. Physical Review A, 2001. 64(4).

20. Wernet, P., et al., Core-valence interactions in the linear dichroism of $\mathrm{Cr} 2 p$ photoelectron spectra. Physical Review B, 2000. 62(21): p. 14331-14336. 
21. Wernet, P., et al., Multiplet splitting and valence-shell recoupling in the corelevel2pphotoelectron spectrum of atomic Mn and of Mn compounds. Physical Review A, 2001. 63(5).

22. Hirsch, K., et al., Initial- and final-state effects on screening and branching ratio in2px-ray absorption of size-selected free3dtransition metal clusters. Physical Review B, 2012. 86(16).

23. Zamudio-Bayer, V., et al., Electronic ground states of Fe2(+) and $\mathrm{Co} 2(+)$ as determined by $x$-ray absorption and $x$-ray magnetic circular dichroism spectroscopy. J Chem Phys, 2015. 143(24): p. 244318.

24. Zamudio-Bayer, V., et al., Maximum spin polarization in chromium dimer cations as demonstrated by X-ray magnetic circular dichroism spectroscopy. Angew Chem Int Ed Engl, 2015. 54(15): p. 4498-501.

25. Wen, A.T., E. Ruhl, and A.P. Hitchcock, Inner-Shell Excitation of Organoiron Compounds by Electron-Impact. Organometallics, 1992. 11(7): p. 2559-2569.

26. Ueda, K., High-resolution inner-shell spectroscopies of free atoms and molecules using soft-Xray beamlines at the third-generation synchrotron radiation sources. Journal of Physics BAtomic Molecular and Optical Physics, 2003. 36(4): p. R1-R47.

27. Heck, A.J.R., et al., Photoionization Dynamics and Charge Separation Reactions of Iron Pentacarbonyl, Enneacarbonyl, and Dodecacarbonyl Complexes Induced by Photoabsorption in the 20-90 Ev Energy-Range. Journal of Physical Chemistry, 1995. 99(42): p. 15633-15641.

28. Sistrunk, E., et al., Resonant photoemission at the iron M-edge of Fe(CO)5. J Chem Phys, 2013. 139(16): p. 164318.

29. Tamenori, Y., B.H. Boo, and I. Koyano, Dissociative photoionization of ferrocene in the $\mathrm{Fe}: 3 p$ inner-valence region. Journal of Electron Spectroscopy and Related Phenomena, 1999. 101: $p$. 87-91.

30. Tamenori, Y. and I. Koyano, Dissociative photoionization of iron pentacarbonyl in the range 38$120 \mathrm{eV}$. Journal of Physical Chemistry A, 1998. 102(2): p. 368-374.

31. Eberhardt, W., et al., Site-Specific Fragmentation of Small Molecules Following Soft-X-Ray Excitation. Physical Review Letters, 1983. 50(14): p. 1038-1041.

32. Eberhardt, W., et al., CORRELATION BETWEEN ELECTRON-EMISSION AND FRAGMENTATION INTO IONS FOLLOWING SOFT-X-RAY EXCITATION OF THE N2 MOLECULE. Physical Review Letters, 1983. 51(26): p. 2370-2373.

33. Miron, C., et al., Site-selective photochemistry of core excited molecules: Role of the internal energy. Physical Review Letters, 1998. 81(19): p. 4104-4107.

34. Nagaoka, S., et al., Site-specific fragmentation caused by core-level photoionization in F3SiCH2CH2Si(CH3)(3) vapor: Comparison between Si:1s and $2 p$ photoionizations by means of photoelectron-photoion-photoion triple-coincidence spectroscopy. Journal of Chemical Physics, 2008. 129(20): p. 7.

35. Ruhl, E., et al., Ionic Fragmentation of Carbon 1s Excited Metallocenes. Chemical Physics, 1993. 169(2): p. 243-257.

36. Guo, M., et al., Simulations of iron $K$ pre-edge $X$-ray absorption spectra using the restricted active space method. Phys Chem Chem Phys, 2016. 18(4): p. 3250-9.

37. Haverkort, M.W., M. Zwierzycki, and O.K. Andersen, Multiplet ligand-field theory using Wannier orbitals. Physical Review B, 2012. 85(16).

38. Ikeno, H., T. Mizoguchi, and I. Tanaka, Ab initiocharge transfer multiplet calculations on the L2,3XANES and ELNES of3dtransition metal oxides. Physical Review B, 2011. 83(15).

39. Josefsson, I., et al., Ab Initio Calculations of X-ray Spectra: Atomic Multiplet and Molecular Orbital Effects in a Multiconfigurational SCF Approach to the L-Edge Spectra of Transition Metal Complexes. J Phys Chem Lett, 2012. 3(23): p. 3565-70.

40. Pinjari, R.V., et al., Restricted active space calculations of L-edge X-ray absorption spectra: from molecular orbitals to multiplet states. J Chem Phys, 2014. 141(12): p. 124116. 
41. Roemelt, M., et al., A combined DFT and restricted open-shell configuration interaction method including spin-orbit coupling: application to transition metal L-edge $X$-ray absorption spectroscopy. J Chem Phys, 2013. 138(20): p. 204101.

42. Kunnus, K., et al., Identification of the dominant photochemical pathways and mechanistic insights to the ultrafast ligand exchange of $\mathrm{Fe}(\mathrm{CO}) 5$ to $\mathrm{Fe}(\mathrm{CO}) 4 \mathrm{EtOH}$. Struct Dyn, 2016. 3(4): p. 043204.

43. Wernet, P., et al., Orbital-specific mapping of the ligand exchange dynamics of $\mathrm{Fe}(\mathrm{CO}) 5$ in solution. Nature, 2015. 520(7545): p. 78-81.

44. Arp, U., et al., $3 d$ photoionization of $\mathrm{Xe}, \mathrm{Cs}$ and $\mathrm{Ba}$ and the collapse of the $4 f$ wavefunction. J. Phys. B: At. Mol. Opt. Phys., 1999. 32: p. 1295-1304.

45. Cramer, S.P., et al., Ligand Field Strengths and Oxidation States from Manganese L-Edge Spectroscopy. J. Am. Chem. Soc., 1991. 113: p. 7937-7940. 